\title{
Comprensión de lectura y velocidad lectora en alumnos de sexto grado de primaria de centros educativos estatales y no estatales de Lima
}

\author{
Miguel Escurra \\ Universidad de Lima \\ Lima - Perú
}

El estudio permitió analizar la relación que existe entre la comprensión de lectura y la velocidad lectora en los alumnos de sexto grado de Lima Metropolitana. Se trabajó con una muestra probabilística de 541 alumnos de los cuales 402 pertenecian a colegios de gestión estatal y 109 a colegios de gestión particular, 272 eran varones y 269 mujeres, entre 11 y 13 años de edad.

La prueba de velocidad lectora presentó validez y confiabilidad.

Las comparaciones indicaron que los alumnos provenientes de colegios particulares presentaron mejores niveles de comprensión de lectura y mayor relación entre la comprensión de lectura y la velocidad lectora que los alumnos de colegios estatales.

lectura / velocidad lectora / comprensión de lectura

\section{Reading understanding and the reading speed in sixth grade students of primary of public and private schools of metropolitan area of Lima-Perú}

The study allowed the analysis of the relationship that exists between reading understanding and reading comprehension and reading speed among sixth grade students in the Metropolitan Area of Lima. The study used a probabilistic sample of 541 students, 402 came from public schools and 109 from private schools, 272 were boys and 269 girls, between 11 to 13 years of age. The reading speed test presented validity and reliability. The comparisons indicated that students from private schools presented better levels of reading comprehension and of reading speed and a greater relationship between reading comprehension and reading speed than that of students from public schools. 


\section{COMPRENSIÓN DE LECTURA}

La comprensión de lectura se puede definir como el proceso por el cual se emplean las claves dadas por el autor y el conocimiento previo que el lector tiene para conocer el significado que aquél intenta transmitir. Es muy importante tener en cuenta que si el conocimiento previo que el lector tiene es sólido y amplio este lector va a construir un modelo de una manera rápida y detallada, entonces la actividad lectora llena huecos en ese modelo y lo verifica (Johnston, 1983; Molina, 1991; Pinzás, 1995; Cuetos, 1996).

Para comprender lo que se lee se utilizan muchas y variadas estrategias. Se parte de unas claves o macroseñales que proporciona el autor y se elabora un modelo. Para comprobar si el modelo corresponde a lo que dice el autor, se analiza si es factible lo que se va asumiendo con la lectura y las consecuencias del modelo. También se analiza lo completo que es un modelo y su interconexión, viendo por último qué correspondencia existe entre el modelo y el texto.

Cuando el modelo no se corresponde con el texto, se ponen en marcha una serie de estrategias para el control de la comprensión, es decir, estrategias de metacomprensión. Se puede fallar al leer, en la comprensión de una palabra, en la comprensión de una o varias frases, o en la comprensión del discurso.

Cuando se controla la comprensión, se necesita conocer las condiciones que desencadenan ese fallo de comprensión. Algunas de las estrategias para salvar estos errores de comprensión, según Smith (1978), son las siguientes:

- Ignorar y seguir leyendo.

- Suspender los juicios.

- Elaborar una hipótesis de tanteo.

- Releer la frase.

- Releer el contexto previo.

- Consultar una fuente experta.

En la actualidad, cuatro son los supuestos básicos que fundamentan la investigación psicológica sobre la comprensión de la lectura:

- La destreza lectora depende de la interacción entre procesos cognitivos, lingüísticos y perceptivos.

- La lectura es un proceso interactivo.

- Nuestra capacidad para procesar información tiene límites.

- La lectura es un proceso estratégico.

Actualmente la destreza lectora se centra en el texto, y la comprensión lectora reside tanto en el lector como en el texto. Al mismo tiempo, el lector aporta sus conocimientos anteriores sobre el contenido y estructura del lenguaje. Es a partir de la publicación del artículo de (Nicasio García, 1995), donde se sugirió la factibilidad de almacenar en la memoria inmediata siete más, menos dos unidades de información, que se despertó el interés por estudiar los problemas de psicolingüística, lo cual se vio reforzado por la teoría de los esquemas de Bruner, las ideas de Chomsky, la antropología cultural y la informática (Bermejo, 1994). 
Los estudios sobre la metacognición han revelado que los lectores son conscientes de su conocimiento y utilizan estrategias para alcanzar sus objetivos (Brown, Armburster \& Baker, 1986; Bermejo, 1994). También el conocimiento que el lector posee puede influir en la comprensión de un texto, al activar éste un esquema interrelacionado.

\section{El ROL DE LOS PROCESOS \\ PERCEPTIVOS Y ATENCIONALES}

Para Wundt, la percepción se produce al combinar los atributos físicos puros e integrarlos para lograr una representación consciente de la experiencia inmediata. La Gestalt, por el contrario, afirma que no percibimos elementos aislados sino globalidades. Desde la psicología cognitiva, la percepción es un proceso a través del cual extraemos información del medio ambiente para adaptarnos a él (Nicasio García, 1995). Además, la percepción ayuda a estructurar el mundo y verlo como algo organizado, de manera que se agrupan los objetos en categorías y se extrae el significado de los estímulos de entrada para formar representaciones que formen el conocimiento perceptual.

Para procesar la información se necesita, en primer lugar, un estímulo (visual) con un determinado nivel de actividad para que se pongan en funcionamiento los receptores visuales que transformen la energía luminosa en energía eléctrica que active al SNC (Sistema Nervioso Central). De mane- ra que cuando el ojo entra en contacto con las palabras, la forma de las letras se convierte en energía nerviosa y se inicia así el proceso de entrada. Una vez ocurrido este proceso la información es almacenada y se activan las pautas de la memoria de corto plazo (MCP) y la memoria de largo plazo (MLP). En este momento se comparan los rasgos extraídos con los ya almacenados, lo que permite el acceso léxico y el reconocimiento de la palabra. Una vez reconocida la palabra se le asigna un significado. Una vez reconocido el estímulo intervienen los procesos atencionales para decidir si el procesamiento continúa o no, tarea que se realiza en la MCP o memoria operativa. Después, las palabras se agrupan en función de reglas sintácticas, produciéndose la codificación de proposiciones. Ya codificadas las proposiciones, se integran en párrafos y si este proceso integrador se ejecuta bien, se activan los esquemas necesarios para dar un significado al texto.

Se han propuesto distintos modelos de reconocimiento de pautas, pero uno de los modelos más relevantes son los basados en la extracción de rasgos, lo que supone seleccionar la información relevante de entrada y compararla con las características almacenadas en la MLP. Para que la información entrante sea identificada es necesario que algunos de sus rasgos destaquen. De esta manera, Gibson (1973) identificó 10 rasgos a partir de los cuales pueden 
representarse casi todas las letras del alfabeto. Algunos de ellos son líneas rectas, curvas, horizontales, verticales, etc., pero los procesos perceptuales y de reconocimiento son más complejos de lo que propone el análisis de rasgos.

Para la psicología cognitiva, el proceso de la lectura y del reconocimiento de palabras se produce de forma global, aceptando el concepto de efecto de superioridad de la palabra, ya que de esta manera es más fácil que se active el conocimiento conceptual. Glass \& Holyoak (Puente, 1991) afirman que las palabras comunes pueden identificarse de forma global, mientras que las desconocidas lo harían a partir de sus elementos. Por otro lado, hay asociaciones en las que la información entrante carece de rasgos llamativos y, en estos casos, adquiere relevancia la atención y el conocimiento previo. Una vez reconocido el patrón de entrada, la persona puede o no prestarle atención. Para explicar cómo se produce la atención se han propuesto diversos modelos: cuello de botella, filtro, entre otros. Neisser (1980) alude a los procesos preatencionales para referir que a veces la atención se produce de forma inconsciente, automática, dependiendo del conocimiento del material, esquemas, riqueza conceptual, etc. De manera que hay diferencias entre lectores expertos, que necesitan menos atención consciente, y los lectores menos expertos, que necesitan más atención consciente. De todas maneras, la atención es limitada y no se puede prestar atención a todos los estímulos.

\section{TEORÍA DEL ESQUEMA Y COMPRENSIÓN DE LECTURA}

Barlett, en 1932 (De Vega, Carreiras, Gutierrez-Calvo \& Alonso-Quecuty, 1990), propuso el concepto de esquema, como un factor que podría explicar procesos complejos como la comprensión y la memoria. En su trabajo sobre recuerdo de textos y su organización en la memoria, llegó a las siguientes conclusiones:

- En el recuerdo de las historias se producen bastantes omisiones.

- Los sujetos utilizan un lenguaje distinto al original.

- El relato es más coherente que el original.

- El texto es adaptado por los lectores desde su propia perspectiva cultural.

De acuerdo con la psicología cognitiva, y con diferentes autores como Anderson (1980) y Rumelhart (1977), entre otros, los esquemas pueden conceptualizarse como:

- Estructuras abstractas que representan lo que uno piensa del mundo.

- Estructuras de datos para representar conceptos generales.

- La unidad de significado y procesamiento del sistema cognitivo.

- Sistemas de representación formados por un conjunto de conocimientos que intervienen en la interpreta- 
ción de datos, recuperación de información organización de la acción, establecimiento de objetivos y metas.

Las características más relevantes de los esquemas según Rumelhart (1977) son:

- Tienen componentes fijos y componentes variables.

- Son de naturaleza holística, pueden incluirse encajando unos dentro de otros.

- Presentan una estructura jerárquica, que representa el conocimiento en todos los niveles de abstracción.

- Representan el conocimiento, tanto declarativo como procedimental.

Rumelhart (1977) indica que los lectores pueden fracasar al comprender un texto por los siguientes motivos:

- No tienen un esquema apropiado para comprender los conceptos.

- El escritor no expresa las claves necesarias para activar los esquemas del lector.

- La interpretación del lector es distinta de la del escritor.

Los supuestos teóricos básicos de la teoría del esquema según McNeil (Bermejo, 1994) son:

- El conocimiento anterior de los niños puede afectar la comprensión de los textos.

- En la comprensión de los textos intervienen tanto procesos de arriba-abajo, como de abajo-arriba.
- Cuanto más profundo es el procesamiento mayor será la comprensión.

- El contexto influye en el proceso de recuerdo.

Anderson (1980), por su parte, propone las siguientes siete funciones del esquema:

- Sirve para asimilar la información del entorno.

- Dirige la atención del sujeto hacia la información nueva.

- Es una estrategia de búsqueda y procesamiento.

- Sirve para hacer inferencias sobre la información que se presenta.

- Organiza los procesos que se tienen que poner en marcha.

- Sirve para hacer resúmenes y realizar síntesis.

- Ayuda a recuperar información de la memoria de largo plazo (MLP).

Como conclusiones sobre la teoría del esquema, en relación con la comprensión de la lectura, se puede indicar que los malos lectores presentan deficiencias de conocimiento, es decir, para comprender un texto hace falta también comprender sus elementos, las palabras. Tienen problemas para relacionar los elementos de un texto, para elaborar e inferir, no tienen conciencia de qué hacer para leer correctamente. Algunos textos; no tienen claves para activar los esquemas adecuados para comprender el texto y finalmente la lectura necesita de la interpretación de los significados. 


\section{ESTRATEGIAS Y HABILIDADES DE LA COMPRENSIÓN DE LECTURA}

Para que el lector pueda realizar una integración de la información, es fundamental que ponga en juego una serie de estrategias y habilidades de lectura, según la demanda de los textos o de la situación.

En general, las estrategias son secuencias integradas de procedimientos de carácter elevado, que se adoptan con un determinado propósito y la planificación de acciones que se desencadenan para lograrlo, así como su evaluación y posible cambio (Solé, 1998, Vidal-Abarca \& Gilabert, 1991).

Vidal-Abarca \& Gilabert (1991) han analizado las estrategias de comprensión que los lectores emplean para comprender la globalidad del texto. Estos autores indican que los lectores hábiles utilizan estrategias eficaces y maduras, que suponen el uso de macrointerpretaciones e inferencias, que le permiten captar el significado global del texto. Ellos, haciendo uso de ciertas habilidades, van elaborando una representación organizada, jerárquica y coherente del contenido del pasaje, teniendo en cuenta no solamente el contenido del texto, sino también los esquemas de conocimiento del propio sujeto.

Los lectores hábiles utilizan tales estrategias de forma inconsciente (Solé, 1998). Por el contrario, los lectores deficientes utilizan estrategias menos eficaces, que no les permiten producir una integración de los diversos contenidos del texto, procesando frase a frase de forma separada y sin relacionar suficientemente el significado del pasaje con sus esquemas de conocimiento previo (Vidal-Abarca \& Gilabert, 1991), lo que correspondería con lo que algunos autores como Alliende \& Condemarín (1994) y Salvia \& Ysseldike (1998) llaman comprensión literal de un texto.

Morales (1997) considera que existe una cantidad ilimitada de estrategias y aunque mucha de ellas son comunes a la mayoría de lectores, existen otras que son de uso muy personal de cada lector y que responden igualmente a características propias de cada uno de ellos. Al respecto, Solé (1998) considera que existen tres tipos de estrategias de comprensión de lectura:

- Las estrategias previas a la lectura, que permiten al lector adquirir objetivos de lectura y actualizar sus conocimientos previos relevantes.

- Las estrategias durante la lectura, que permiten establecer inferencias de distinto tipo, revisar y comprobar la propia comprensión mientras se lee y tomar acciones adecuadas ante errores o fallos en la comprensión.

- Las estrategias durante/después de la lectura, dirigidas a recapitular el contenido, a resumirlo y a extender el conocimiento que mediante la lectura se ha obtenido.

Uno de los factores más importantes y que asegura el uso adecuado de estas 
estrategias es el conocimiento de ellas, lo que se conoce como metacognición, es decir, el conocimiento acerca de los propios procesos de pensamiento (Pinzás, 1995, 1997, Vidal-Abarca \& Gilabert, 1991). Esto supone que el lector sea capaz de guiar y monitorear su propia lectura. En ese sentido, todas las estrategias de comprensión que el lector utiliza son metacognitivas, permitiéndole intensificar su comprensión y el recuerdo de lo que lee, así como detectar y compensar los posibles errores o fallos de comprensión. Estas estrategias son las responsables de que pueda construirse una interpretación del texto y que el lector sea consciente de qué entiende y qué no entiende para proceder a solucionar el problema encontrado. Para llevar a cabo estas estrategias de comprensión, se necesitan una serie de habilidades básicas, según lo requiere el lector, el texto o la situación de lectura.

\section{FACTORES RELACIONADOS CON LA COMPRENSIÓN DE LECTURA}

La comprensión de lectura es un fenómeno muy complejo y como tal los factores que influyen en ella son también numerosos, están mezclados entre sí y cambian constantemente ( Samuels \& Kamil, 1984; Alliende \& Condemarín 1994).

Thorne \& Pinzás (1988) consideran que la lectura puede estar influeida por un conjunto de variables como la edad, las habilidades, los materiales usados, el tipo de tarea, la interacción profesoralumnos y la clase donde interactúan, entre otros. Para Pinzás (1987), éstos constituyen factores internos, los cuales tienen una influencia directa sobre la lectura. Sin embargo, existe un segundo grupo cuya influencia es indirecta y no por ello menos importante que la autora denominó factores externos. Dentro de este grupo se encuentran la filosofía educativa del país, el currículo, los antecedentes educacionales de los profesores, los sistemas de educación magisterial, el presupuesto asignado, las características culturales, socioeconómicas, psicosociales y lingüísticas de la población, entre otros. Estos factores tienen gran importancia en el desarrollo de la lectura dentro de un país, pues van a tener un efecto significativo sobre los factores internos.

Greaney (1996) encuentra que en los países en vías de desarrollo existe una gran mayoría de personas que no logra alcanzar niveles adecuados de lectura y relaciona estos hallazgos con factores culturales y socioeconómicos que determinan situaciones adversas en el hogar y la escuela, los cuales afectan negativamente el desarrollo de la lectura.

\section{VELOCIDAD LECTORA}

La velocidad lectora puede ser definida como la rapidez de lectura que presentan los sujetos durante un determinado período de tiempo (Fry, 1975).

Nuttall (1996) describe la frustración que produce el leer lentamente, en su 
descripción del círculo vicioso del lector débil. Los lectores que no entienden un material a menudo disminuyen la tasa de lectura y entonces no les gusta leer porque les toma mucho tiempo. Como resultado, ellos leen poco y así continúa el círculo vicioso. Nuttall sugiere que aumentando las proporciones de lectura, el lector pueda entrar en el círculo del buen lector. $\mathrm{Al}$ leer más rápidamente se anima, y con más lecturas la comprensión mejora.

Este concepto de Nuttall se apoya en la propuesta de Stanovich (1980), quien alude a un fenómeno identificado por Merton (1968, en Bermejo, 1994) como el "efecto de Matthew", el cual sugiere que los estudiantes que más leen, aumentan sus habilidades de lectura.

Para Stanovich (1980), los lectores que están leyendo bien y que tienen un buen vocabulario leerán más, y aprenderán más significados. Por el contrario, los lectores con vocabularios inadecuados que leen despacio, tienden a leer menos, lo cual da como resultado que ellos tengan un desarrollo más lento de su vocabulario, lo que a su vez inhibe el desarrollo de la habilidad lectora.

Fry (1975), distingue tres niveles de velocidad lectora, según el tipo de lectura que se realiza:

- Lectura corriente y común: Es la empleada para la lectura diaria de revistas, libros y textos fáciles. Normalmente se desarrolla a una velocidad de 250-500 palabras por mi- nuto (ppm) y con un nivel comprensivo de aproximadamente $70 \%$.

- Lectura de estudio: Es la más lenta y se emplea normalmente con materiales más difíciles y exige un alto nivel de concentración y atención, debiendo ser su nivel comprensivo muy alto. Por lo general se desarrolla a una velocidad de 180-200 palabras por minuto y alcanza un nivel comprensivo de aproximadamente un $90 \%$.

- Lectura superficial: Es aquella que se utiliza para explorar textos, bien porque es el objetivo, bien porque no hay más tiempo. Normalmente su nivel comprensivo se halla situado en el $50 \%$ y su velocidad puede superar las $800 \mathrm{ppm}$.

Existen datos contradictorios con respecto a la proporción óptima de lectura. Algunos autores sugieren que 180 palabras por minuto puede ser un umbral entre la lectura inmadura y la lectura madura y que una velocidad debajo de esto límite es demasiado lenta para lograr una comprensión eficaz (Higgins \& Wallace, 1989). Dubin \& Bycina (1991) indican que una proporción de 200 palabras por minuto parecería ser el mínimo absoluto para leer con adecuada comprensión.

Jensen (1986) y Nuttall (1996), por su parte, establecen que una lectura de 300 palabras por minuto es una proporción óptima. En tanto que Espada (2000) indica que para sexto grado 110 
palabras por minuto es una proporción adecuada. Los principales factores que influyen en el desarrollo de una lectura lenta son:

- Leer todo a la misma velocidad: Algunos materiales son más difíciles de comprender que otros. Hay que saber adaptar la velocidad de la lectura al nivel de dificultad del texto.

- Movimientos ineficientes de los ojos: Lo cual incluye lo siguiente:

- Regresiones: Son las vueltas atrás que se suelen realizar al estar leyendo. El 90\% de ellas son habituales, innecesarias e inconscientes. Las regresiones, hechas de manera intencionada no son motivo de preocupación por que a veces son esenciales para la comprensión.

- Movimientos arrítmicos: Son los movimientos que interrumpen la fluida progresión de fijaciones (momento en el que los ojos se detienen para leer) y barridas de retorno (cuando se pasa de una línea a otra). Es decir, cuando se lee sin "ritmo".

- Barridas de retorno defectuosas: Se produce cuando se pasa de una línea a otra, y se tiene la tentación de "vagar" en vez de pasar directamente a la primera palabra de la siguiente línea.

- Poca superficie de fijación: Los ojos perciben entre 3 y 4 palabras cada vez que se fijan. El pequeño número de palabras que se mira es consecuencia de la manera en que se enseña al niño a leer, palabra por palabra.

- Subvocalización: Consiste en decirse las palabras según se va leyendo. Esto es lo que se llama reafirmación auditiva. Éste es un mal hábito adquirido cuando el profesor hace leer al niño en voz alta para asegurarse de que está aprendiendo la relación entre las letras y los sonidos. La clave está en conseguir leer sin "decirse" las palabras, ya que al hacerlo se limita la velocidad de lectura a la velocidad con la que se habla.

- Baja concentración: La concentración es la capacidad de mantener la atención. Las distracciones internas y externas son verdaderas "asesinas" de la comprensión y la motivación.

- Memoria: Al leer despacio se fragmenta el material de lectura, es decir, limita la perspectiva de lo leído. Leer rápido permite captar conceptos e ideas con claridad.

La lectura constituye en el proceso educativo un elemento básico para el desarrollo del aprendizaje del alumno, debido a que es uno de los principales medios básicos a través de los cuales los alumnos adquieren nuevos conocimientos. En este contexto la velocidad lectora y la comprensión de lectura cumplen un rol muy importante, pues permiten al alumno mejorar la eficacia lectora y entender todo lo que lee con 
rapidez, lo cual a su vez posibilitaría el posterior desarrollo de un conocimiento más completo de su realidad.

Gates, en 1921, determinó que la comprensión de lectura y la velocidad lectora son dos factores distintos pero relacionados, que deben ser incluidos en un programa de lectura. Por su parte, LaBerge \& Samuels (1985) desarrollaron esta idea en su modelo, dando énfasis a la importancia de la automaticidad de ciertos componentes del proceso de lectura. Básicamente, ellos teorizan que los lectores que pueden identificar las palabras rápidamente ("automáticamente”) comprenderán bien, debido a que ellos pueden consagrar la mayoría de su atención a la comprensión, es decir, los significados y relaciones de esas palabras.

El debate que rodea la relación real entre estos dos factores ha durado más de la mitad de un siglo. Pues los autores se han preguntado si son totalmente independientes, están relacionados o existe entre ellos una relación de causa y efecto.

Para Nuttall (1996) y Champeau de López (1993), la velocidad lectora y la comprensión de lectura están estrechamente relacionados, pero la naturaleza precisa del eslabón entre ellos ha sido el enfoque de un debate. Se piensa que un lector lento muy probablemente tendrá una baja comprensión de lectura, debido a que su memoria realiza un mayor esfuerzo debido a la incapacidad para retener la información en trozos pequeños pero lo suficientemente grandes como para progresar a través de un texto con la retención adecuada del volumen en el mensaje. De tal modo que antes de llegar al final de la página, o incluso de una frase, el sujeto ya se olvidó lo que ha leído al principio.

El análisis de las investigaciones realizadas en el Perú con respecto a la lectura, la comprensión de lectura y la velocidad lectora, muestra que existen diferentes investigaciones desarrolladas por Thorne (1991), Pinzás (1986; 1993 ), Noriega (1998), Tapia (1999) y Carreño (2000), quienes indican que la lectura y la comprensión lectora son aspectos que no se han desarrollado adecuadamente ni en el nivel primario ni en el secundario.

Este hallazgo es corroborado en el nivel adulto en el estudio realizado por Gonzales (1998) en estudiantes universitarios, al identificar que el problema de comprensión lectora subsiste y se puede notar una gran incidencia del llamado analfabetismo funcional, es decir, que el sujeto sabe leer y escribir pero tiene una pobre comprensión lectora.

Si se considera que la adquisición de la lectura se inicia en los primeros grados, es de suponer que la eficacia lectora que incluye la comprensión de lectura y la velocidad lectora debería estar consolidada al finalizar la educación primaria, es por ello que se propone estudiar si existe relación entre la comprensión de lectura y la velocidad lectora en los alumnos del sexto grado de 
primaria provenientes de colegios estatales y particulares de Lima Metropolitana.

\section{MÉTodo}

La presente investigación es de tipo correlacional (Graziano \& Raulin, 1989; Alarcón, 1991; Rosenberg \& Daly, 1993; Rosnow \& Rosenthal, 1996; Goodwin, 1998; Hernández, Fernández \& Baptista, 1998; Sánchez \& Reyes, 1998; Salkind, 2000), debido a que permite la recolección de evidencias de hechos concentrándose en tomar medidas e indagar por supuestas relaciones entre ellas, como es el caso de la comprensión de lectura y la velocidad lectora.

El estudio se realizó en dos momentos. En primer lugar se utilizó el diseño correlacional simple (Alarcón,1991; Sánchez \& Reyes, 1998), pues se trata de establecer la relación entre la comprensión lectora y la velocidad lectora. $\mathrm{Su}$ esquema es

$\begin{array}{cc} & \mathrm{O}_{1} \\ \text { Muestra } & \mathrm{r} \\ & \mathrm{O}_{2}\end{array}$

Donde:

$\mathrm{O}_{1}=$ Comprensión de lectura.

$\mathrm{O}_{2}=$ Velocidad lectora

En segundo lugar, se utilizó el diseño descriptivo comparativo de grupos (Alarcón, 1991, Hernández, Fernández \& Baptista, 1998; Sánchez \& Reyes, 1998), pues se trató de comparar si existían diferencias entre las relaciones existentes entre las variables dependientes y la variable independiente asignada, que constituyeron el tipo de gestión del colegio. Su esquema es el siguiente.

$\begin{array}{lll}\text { Grupo } 1 & \mathrm{O}_{1} & \mathrm{O}_{2} \\ \text { Grupo } 2 & \mathrm{O}_{1} & \mathrm{O}_{2}\end{array}$

Donde:

Grupo 1 = Colegio de gestión estatal Grupo 2 = Colegio de gestión particular.

$\mathrm{O}_{1}=$ Comprensión de lectura .

$\mathrm{O}_{2}=$ Velocidad lectora.

\section{Población y muestra}

La población estuvo constituida por los alumnos de sexto grado de educación primaria. De acuerdo con el informe estadístico (2001) del Ministerio de Educación, existen 16.289 casos, los cuales están agrupados según el tipo de colegio en que estudian y la USE a la que pertenecen; se distribuyen conforme al cuadro $\mathrm{N}^{\circ} 1$.

La distribución de los alumnos según tipo de colegio y sexo se presenta en el cuadro $\mathrm{N}^{\mathrm{o}} 2$.

\section{Diseño muestra}

El tamaño de la muestra de investigación fue establecido a través del programa informático Sotam, el cual determinó que, con un margen de error del $5 \%$ y un nivel de confianza del $99,0 \%$, el tamaño de la muestra es de 541 casos. 


\section{Cuadro $N^{\circ} 1$ \\ Composición de la población de alumnos del sexto grado de primaria por USE de Lima Metropolitana}

\begin{tabular}{lcccccc}
\hline USE & Estatal & Particular & Total & $\begin{array}{c}\% \\
\text { Estatal }\end{array}$ & $\begin{array}{c}\% \\
\text { Particular }\end{array}$ & $\begin{array}{c}\% \\
\text { Total }\end{array}$ \\
\hline USE 1 & 1053 & 294 & 1347 & 6,47 & 1,80 & 8,27 \\
USE 2 & 2460 & 537 & 2997 & 15,10 & 3,30 & 18,40 \\
USE 3 & 1975 & 601 & 2576 & 12,13 & 3,69 & 15,81 \\
USE 4 & 1085 & 817 & 1902 & 6,66 & 5,01 & 11,68 \\
USE 5 & 1654 & 438 & 2092 & 10,16 & 2,69 & 12,84 \\
USE 6 & 1524 & 418 & 1942 & 9,36 & 2,56 & 11,92 \\
USE 7 & 1289 & 355 & 1644 & 7,91 & 2,18 & 10,09 \\
USE 8 & 1069 & 720 & 1789 & 6,56 & 4,42 & 10,98 \\
Total & 12109 & 4180 & 16289 & 74,35 & 25,65 & 100,00 \\
\hline
\end{tabular}

Fuente: Ministerio de Educación, 2001

\section{Cuadro $\mathrm{N}^{\circ} 2$}

Composición de la población por género y tipo de colegio

\begin{tabular}{|c|c|c|c|}
\hline \multirow[t]{2}{*}{ Tipo de colegio } & \multicolumn{2}{|c|}{ Género } & \multirow[t]{2}{*}{ Total } \\
\hline & Varón & Mujer & \\
\hline \multirow[t]{2}{*}{ Estatal } & 6473 & 6405 & 12878 \\
\hline & $39,74 \%$ & $39,32 \%$ & $79,06 \%$ \\
\hline \multirow[t]{2}{*}{ Particular } & 1663 & 1748 & 3411 \\
\hline & $10,21 \%$ & $10,73 \%$ & $20,94 \%$ \\
\hline \multirow[t]{2}{*}{ Total } & 8136 & 8153 & 16289 \\
\hline & $49,95 \%$ & $50,05 \%$ & $100,00 \%$ \\
\hline
\end{tabular}

Fuente: Ministerio de Educación, 2001

\section{Muestreo}

Dada la naturaleza del estudio se desarrolló un muestreo probabilístico por conglomerados bi-etápico (Lohr, 1999; Pérez, 2000), de acuerdo con el siguiente procedimiento:

En la primera etapa, se construyó el marco muestral (Babbie, 2000) con el listado de colegios organizados por USE, presentado en la base de datos del Ministerio de Educación del 2001. En este momento se utilizó un muestreo por conglomerados, siendo las USE las unidades más generales de agrupamiento a partir de las cuales se eligieron de manera aleatoria los colegios que participaron en el estudio.

En la segunda etapa, en cada colegio seleccionado se eligieron de forma aleatoria las aulas de sexto grado del 


\section{Cuadro $\mathrm{N}^{\circ} 3$}

Composición de la muestra de alumnos por USE

\begin{tabular}{lcccccc}
\hline USE. & Estatal & Particular & Total & $\begin{array}{c}\% \\
\text { Estatal }\end{array}$ & $\begin{array}{c}\% \\
\text { Particular }\end{array}$ & $\begin{array}{c}\% \\
\text { Total }\end{array}$ \\
\hline USE 1 & 30 & 8 & 38 & 35 & 10 & 45 \\
USE 2 & 76 & 16 & 92 & 82 & 18 & 100 \\
USE 3 & 60 & 19 & 79 & 66 & 20 & 86 \\
USE 4 & 31 & 26 & 57 & 36 & 27 & 63 \\
USE 5 & 50 & 13 & 63 & 55 & 15 & 69 \\
USE 6 & 45 & 12 & 57 & 51 & 14 & 64 \\
USE 7 & 38 & 10 & 48 & 43 & 12 & 55 \\
USE 8 & 30 & 23 & 53 & 36 & 24 & 59 \\
Total & 360 & 127 & 487 & 73.92 & 26.08 & 100 \\
\hline
\end{tabular}

Fuente: Ministerio de Educación, 2001

nivel primario que se incluyeron en el estudio. En cada aula se incluyeron en el estudio, previa conformidad, sólo aquellos alumnos que presentaron una edad cronológica entre 10, 11 y 12 años de edad cumplidos al momento de la evaluación. A continuación, se presenta la proporción y tamaño de muestra de los alumnos que fueron requeridos por USE (cuadro $\mathrm{N}^{\circ} 3$ ).

Resulta necesario destacar que la representatividad de la muestra se realizó sobre la base del número de alumnos por USE y no al número de colegios, debido a que el número de colegios particulares es mayor que el de los colegios estatales, pues en el nivel de los centros la distribución es totalmente inversa.

\section{Composición de la muestra}

Las principales características sociodemográficas de la muestra de alumnos evaluados son presentadas en los siguientes cuadros.

\section{Cuadro $\mathrm{N}^{\circ} 4$}

Composición de la muestra por género y tipo de colegio

\begin{tabular}{lccc}
\hline $\begin{array}{l}\text { Tipo de } \\
\text { colegio }\end{array}$ & \multicolumn{2}{c}{ Género } & Total \\
\hline \multirow{4}{*}{ Estatal } & Varón & Mujer \\
& 202 & 200 & 402 \\
Particular & $37,33 \%$ & $36,97 \%$ & $74,30 \%$ \\
& 70 & 69 & 139 \\
Total & $12,93 \%$ & $12,75 \%$ & $25,70 \%$ \\
& $272 \%$ & $269 \%$ & $541 \%$ \\
& $50,27 \%$ & $49,72 \%$ & $100,00 \%$ \\
\hline
\end{tabular}

El análisis de la composición de la muestra indica que el $74,30 \%$ corresponden a los alumnos provenientes de colegios estatales y por el $25,70 \%$ a colegios particulares. La composición por sexo indica que el $50,30 \%$ son varones y el $49,70 \%$ son mujeres. 


\begin{tabular}{|c|c|c|}
\hline \multicolumn{3}{|c|}{$\begin{array}{c}\text { Cuadro } \mathrm{N}^{\circ} 5 \\
\text { Composición general de la muestra } \\
\text { de alumnos evaluados por edad }\end{array}$} \\
\hline Edad & $\mathrm{F}$ & $\%$ \\
\hline 11 años & 228 & 42.15 \\
\hline 12 años & 248 & 45.84 \\
\hline 13 años & 65 & 12.01 \\
\hline Total & 541 & 100.00 \\
\hline
\end{tabular}

El análisis de la composición de la muestra por edad (cuadro $\mathrm{N}^{\circ} 5$ ) indica que se presentan alumnos con edades entre 11 y 13 años, de los cuales la mayoría corresponde a los que tienen 12 años $(45,84 \%)$, seguida por los que tienen 11 años de edad $(42,15 \%)$ y por los que tienen 13 años (12,01\%).

\section{Instrumento}

\section{Prueba de comprensión de lectura para sexto grado (PCL-6)}

Para el desarrollo del presente estudio se utilizó la prueba de comprensión de lectura para sexto grado (PCL-6), elaborada por Carreño (2000), la cual permite determinar el nivel de logro de los estudiantes teniendo en cuenta las exigencias curriculares del sexto grado y los niveles esperados para niños de esas edades de acuerdo con la literatura especializada.

La prueba de comprensión lectora está constituida por dos textos (ver anexo), cada uno de los cuales va seguido por preguntas con respuestas de opción múltiple. Las cuales permiten evaluar:
- El nivel de comprensión literal del texto: explora el entendimiento de hechos y detalles que aparecen en el texto. Está constituido por 10 preguntas que incluyen los ítemes 1,2 , $3,4,5$ y 6 de la primera parte y los ítemes 2, 3, 4 y 5 de la segunda parte.

- El nivel de comprensión inferencial del texto: evalúa la deducción de información. Está conformado por 18 preguntas, que incluye los ítemes 7 , $8,9,10,11,12,13,14$, y 15 de la primera parte y los ítemes $1,6,7,8$, $9,10,11,12$, y 13 de la segunda parte.

Los textos utilizados son de tipo narrativo y presentan una historia con hechos que transcurren en un orden temporal determinado, fácil de visualizar y presentan personajes concretos e individuales.

El primer criterio utilizado para elaborar los textos fue su longitud, medidos a través del número de palabras $\mathrm{y}$ oraciones, tomando como referencia la longitud de algunos textos escolares para sexto grado y la longitud de los textos de la prueba de complejidad lingüística progresiva de Alliende, Condemarín y Milicic (1993).

La prueba de comprensión de lectura, presentó validez de contenido establecida de acuerdo con el criterio de jueces (Carreño, 2000). Así como también validez de constructo establecida a partir de una serie de estudios que demostraron tener concordancia con la natu- 
raleza del constructo estudiado. Los resultados demostraron que la prueba era capaz de diferenciar a los buenos de los malos lectores, y que existía un mejor desempeño en las preguntas de comprensión literal que en las preguntas de comprensión inferencial (Carreño, 2000).

La confiabilidad fue estimada a través del procedimiento de la consistencia interna sobre la base de los coeficientes Kuder Richardson 20. Al trabajarse en una muestra de 3.354 alumnos se alcanzó un valor de 0.89 para toda la muestra, de 0.77 para la primera parte y de 0.76 para la segunda parte. Estos resultados demostraron que la prueba permite obtener puntajes confiables (Carreño, 2000).

En el desarrollo del presente estudio se consideró pertinente siguiendo a Anastasi (1986), volver a revisar la validez y confiabilidad del instrumento con el fin de garantizar la adecuación psicométrica de la prueba, encontrándose los resultados que se detallan a continuación.

\section{Análisis psicométrico}

La validez del instrumento fue estudiada a través de la validez de constructo, por medio del método intraprueba (Brown, 1996; Anastasi \& Urbina, 1998), efectuándose el análisis factorial confirmatorio de manera que se tra- tó de establecer si los dos tipos de comprensión (literal e inferencial) correspondían a una sola dimensión en los datos de la muestra trabajada. Los resultados que se dan a conocer en el gráfico $\mathrm{N}^{\mathrm{o}} 1$ y el cuadro $\mathrm{N}^{\circ} 6$, permiten observar que el modelo de 1 factor alcanza mejores resultados que el modelo independiente de manera que se observa un estadístico chi-cuadrado (3.50) que no es significativo, a la vez que el análisis de residuales indica que los valores son inferiores al criterio de 0.05 , y también que los estadísticos encargados de evaluar la adecuación del modelo (GFI, AGFI) son adecuados, por lo que se concluye que el modelo es corroborado en los datos evaluados y por lo tanto la prueba de comprensión lectora para sexto grado (PCL-6) presenta validez de constructo.

\section{Cuadro $\mathrm{N}^{\circ} 6$}

Análisis factorial confirmatorio de la prueba de comprensión de lectura para sexto grado (PCL-6)

\begin{tabular}{lcc}
\hline Datos & $\begin{array}{c}\text { Modelo } \\
\text { de 1 Factor }\end{array}$ & $\begin{array}{c}\text { Modelo } \\
\text { independiente }\end{array}$ \\
\hline Parámetros & 8 & 4 \\
Chi-cuadrado & 3.50 & 1107.476 \\
mínimo & & \\
G. L. & 2 & 6 \\
P & .650 & .000 \\
Chi-cuadrado & 1.75 & 184.579 \\
mínimo / G. L. & & \\
RMR & 0.033 & 2.211 \\
GFI & 0.991 & 0.487 \\
AGFI & 0.995 & 0.390 \\
\hline $\mathrm{N}=541$ & &
\end{tabular}




\section{Gráfico $\mathbf{N}^{\circ} 1$ \\ Modelo del análisis factorial confirmatorio del PCL-6}

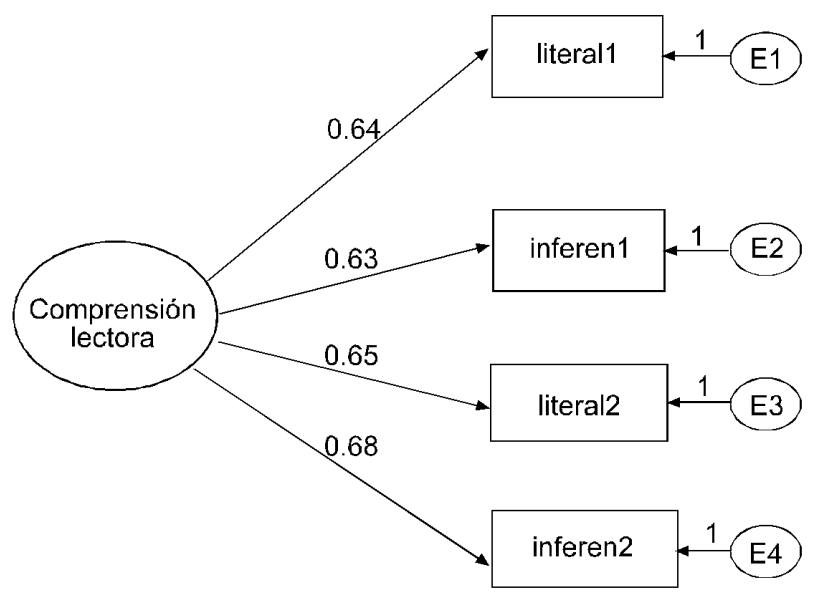

Análisis factorial confirmatorio del PCL-6

Chi - cuadrado $=3.50$

G. L. $=2$

$p=.620$

Para estudiar la confiabilidad se utilizó el análisis de ítemes y el método de la consistencia interna a través del coeficiente Kuder Richardson 20. El análisis de la prueba completa alcanzó un valor de 0.94 , por lo que se puede concluir que la prueba permite obtener puntajes confiables. A continuación se presenta el análisis de cada parte.

El análisis de los ítemes de la primera parte de la prueba de comprensión de lectura para sexto grado (PCL-6) presentado en el cuadro $\mathrm{N}^{\circ} 7$, permite apreciar que todos los ítemes alcanzan correlaciones significativas, notándose además que dichos valores oscilan entere 0.21 y 0.65 , lo que permite retener

\section{Cuadro $\mathrm{N}^{\circ} 7$}

Análisis de ítemes y confiabilidad de la primera parte de la prueba de comprensión de lectura de sexto grado (PCL-6)

\begin{tabular}{cccc}
\hline Item & $\mathrm{M}$ & $\mathrm{D} . \mathrm{E}$. & .ritc \\
\hline 1 & 0.86 & 0.13 & $0.21^{*}$ \\
2 & 0.73 & 0.26 & 0.22 * \\
3 & 0.85 & 0.36 & $0.30^{*}$ \\
4 & 0.80 & 0.40 & $0.611^{*}$ \\
5 & 0.83 & 0.26 & $0.35^{*}$ \\
6 & 0.86 & 0.34 & 0.22 * \\
7 & 0.83 & 0.37 & $0.38^{*}$ \\
8 & 0.77 & 0.42 & $0.36^{*}$ \\
9 & 0.38 & 0.49 & $0.41^{*}$ \\
10 & 0.65 & 0.48 & 0.59 * \\
11 & 0.71 & 0.71 & 0.62 * \\
12 & 0.58 & 0.58 & 0.60 * \\
13 & 0.55 & 0.55 & 0.58 * \\
14 & 0.68 & 0.47 & 0.65 * \\
15 & 0.73 & 0.44 & 0.61 * \\
\hline
\end{tabular}

Kuder Richardson $20=0.80$ *

${ }^{*} p<.05$

$\mathrm{N}=541$ 
todos los ítemes de la prueba. Respecto de la confiabilidad también se puede apreciar que el coeficiente Kuder Richardson 20 asciende a 0.80 lo que garantiza que el instrumento permite obtener puntajes confiables.

\section{Cuadro $\mathbf{N}^{\circ} 8$}

Análisis de ítemes y confiabilidad de la segunda parte de la prueba de comprensión lectora de sexto grado (PCL-6)

\begin{tabular}{cccc}
\hline Item M & D. E. & .ritc & \\
\hline 1 & 0.71 & 0.46 & 0.39 * \\
2 & 0.85 & 0.35 & $0.35^{*}$ \\
3 & 0.53 & 0.50 & $0.40^{*}$ \\
4 & 0.72 & 0.45 & $0.64^{*}$ \\
5 & 0.72 & 0.45 & $0.75^{*}$ \\
6 & 0.72 & 0.45 & $0.71^{*}$ \\
7 & 0.62 & 0.49 & $0.65^{*}$ \\
8 & 0.52 & 0.50 & $0.62^{*}$ \\
9 & 0.70 & 0.46 & 0.72 * \\
10 & 0.50 & 0.50 & 0.61 * \\
11 & 0.39 & 0.49 & $0.57^{*}$ \\
12 & 0.52 & 0.50 & 0.64 * \\
13 & 0.64 & 0.48 & 0.70 * \\
& & & \\
\hline
\end{tabular}

Kuder Richardson $20=0.90$ *

$* p<.05$

$\mathrm{N}=541$

En lo que concierne al análisis de los ítemes de la segunda parte de la prueba de comprensión de lectura, presentado en el cuadro $\mathrm{N}^{\circ} 8$, permite notar que todos los ítemes alcanzan correlaciones significativas, apreciándose además que dichos valores oscilan entre $0.35 \mathrm{y}$ 0.75 , lo que permite retener todos los ítemes de la prueba. Respecto de la confiabilidad también se puede apreciar que el coeficiente Kuder Richard- son 20 asciende a 0.90 , lo que garantiza que el instrumento permite obtener puntajes confiables.

\section{Prueba de Velocidad lectora (PVL-2000)}

Para evaluar la velocidad de lectura se utilizó como instrumento el texto adaptado por Espada (2000), cuya longitud es más larga de lo que el niño puede leer en un minuto. El alumno debe leer durante un minuto la prueba respectiva. En el anexo 2 se presenta la lectura correspondiente.

Si el alumno tiene un error se le pide que lo repita y corrija, si no logra leerlo correctamente al tercer intento, se le dice la palabra y se permite que continúe leyendo.

Cuando el alumno repite la misma palabra o retrocede en la lectura, se consideran errores y no aumentan las palabras leídas, aunque suponen un tiempo perdido que se reflejará en el menor número de palabras leídas.

Los puntos, comas, signos de admiración o interrogación y guiones no están contados, si se da el caso de que un alumno termina la lectura antes del minuto deberá empezar de nuevo el texto hasta completar el minuto y sumar las palabras hasta donde haya llegado. El título de la lectura también debe leerse.

El análisis estadístico de las características de la lectura indica que en términos de legibilidad presentaba un nivel intermedio de $50 \%$ en la escala de Flesch, con un $48 \%$ de complejidad 
oracional y un $60 \%$ de complejidad de vocabulario.

El análisis de la lectura indica que presentó: 371 caracteres, 568 sílabas, 289 palabras, 17 oraciones, 5 párrafos, 7 oraciones cortas, 4 oraciones largas, 6 oraciones simples.

La validez de la prueba de velocidad lectora se realizó a través de la validez de contenido, la cual tiene por finalidad determinar el grado con que una prueba representa el universo de contenido del cual proviene (Brown, 1996). Para ello se aplicó el método del criterio de jueces, se encargó a 10 profesionales, expertos psicólogos educacionales, que evaluaran la prueba, analizando las respuestas a través del coeficiente $\mathrm{V}$ de Aiken (Escurra, 1988). Los resultados indicaron que se obtuvo un coeficiente de 0.95 , hallazgo que permite concluir que la prueba tiene validez de contenido.

Debido a la naturaleza de la prueba, se trabajó la confiabilidad test-retest, que consiste en evaluar una muestra de sujetos con el mismo instrumento en dos ocasiones diferentes para determinar la estabilidad de los resultados. Se aplicó la misma prueba a una muestra de 50 alumnos con un intervalo de 3 semanas, encontrándose un coeficiente de correlación de Pearson de 0.89, hallazgo que corrobora que la prueba permite obtener puntajes confiables.

\section{Sistema de calificación}

Para calcular la cantidad de palabras leídas por minuto se hace leer al niño y se toma en cuenta el tiempo que demora en completarla, para ello se usa un cronómetro y se calcula en segundos. Tomando en cuenta la cantidad de palabras que tiene la lectura se aplica la siguiente fórmula:

$$
\text { Ppm = } \quad \begin{aligned}
& \text { Número de palabras que tiene } \\
& \text { la lectura multiplicado por } 60
\end{aligned}
$$

\section{Procedimiento de recolección de datos}

Para la recolección de datos se utilizó el siguiente procedimiento: en primer lugar se establecieron con los colegios seleccionados las coordinaciones respectivas para realizar las evaluaciones. En conformidad con los resultados obtenidos, y siguiendo un procedimiento de muestreo aleatorio, se eligieron las aulas de sexto grado a ser evaluadas.

Una vez seleccionadas las aulas, se procedió a elegir los alumnos que habían estudiado el año 2000 en el centro y que tenían entre 10 y 12 años de edad cumplidos al momento de la evaluación. Luego se realizaron las coordinaciones necesarias para aplicar los instrumentos respectivos.

La aplicación de la prueba de comprensión de lectura se realizó de forma grupal y se tomó en cuenta sólo a los alumnos presentes en el momento de la evaluación. La aplicación de la prueba de velocidad lectora fue realizada el mismo día y de forma individual. Las 
evaluaciones se realizaron entre agosto y octubre del 2001.

\section{Resultados}

\section{Análisis descriptivo de las variables}

El análisis descriptivo de toda la muestra, presentado en el cuadro $\mathrm{N}^{\circ}$ 9, permite observar que en el caso de la comprensión literal el valor de la moda $(\mathrm{Mo}=10)$ supera al valor de la media $(\mathrm{M}=8.17)$ y la mediana $(\mathrm{Md}=8.00)$. También se encuentra una desviación estándar pequeña (D. E. = 1.67), lo que indica que el grupo presenta un adecuado nivel de homogeneidad.

En el análisis de la comprensión inferencial también se encuentra que la moda $($ Mo $=18.00)$ es mayor que la mediana $(\mathrm{Md}=12.00)$ y la media $(\mathrm{M}=$ 11.20), presentando una desviación estándar media (D. E. = 5.22), por lo que se concluye que el grupo presenta un moderado nivel de homogeneidad.

En los puntajes de la comprensión de lectura, se observa que la mediana (Md $=20.00)$ es mayor que la media $(\mathrm{M}=$ 19.37) y la moda (Mo $=19.00)$, presentando una desviación estándar media (D. E. =6.59), por lo que se concluye que el grupo presenta un moderado nivel de homogeneidad.

El análisis de la velocidad lectora permite apreciar que la mediana $(\mathrm{Md}=$ $39.00)$ es menor que la media $(\mathrm{M}=$
42.81) y la moda $(\mathrm{Mo}=66.00)$. Además se observa que presenta una desviación estándar alta (D.E. $=20.86$ ), por lo que se concluye que el grupo presenta un bajo nivel de homogeneidad.

\section{Cuadro $\mathrm{N}^{\circ} 9$}

Análisis descriptivo de las variables estudiadas de toda la muestra

\begin{tabular}{lrccc}
\hline Variable & Media & Mediana & Moda & D.E. \\
\hline $\begin{array}{l}\text { Comprensión } \\
\text { literal }\end{array}$ & 8.17 & 8.00 & 10.00 & 1.67 \\
$\begin{array}{l}\text { Comprensión } \\
\text { inferencial }\end{array}$ & 11.20 & 12.00 & 18.00 & 5.22 \\
$\begin{array}{l}\text { Comprensión } \\
\text { de lectura }\end{array}$ & 19.37 & 20.00 & 19.00 & 6.59 \\
$\begin{array}{l}\text { Velocidad } \\
\text { lectora }\end{array}$ & 42.81 & 39.00 & 66.00 & 20.86 \\
& & & &
\end{tabular}

$\mathrm{N}=541$

Los resultados del análisis de la bondad de ajuste a la curva normal, realizado a través de la prueba de Kolmogorov-Smirnov (cuadro $\mathrm{N}^{\mathrm{o}} 10$ ) indican que en la comprensión literal, la comprensión inferencial, la comprensión de lectura y la velocidad lectora se obtienen estadísticos (K-S Z) que presentan significación estadística, por lo que se puede concluir que las distribuciones de las variables analizadas no se aproximan a la distribución normal. Es debido a estos resultados que los análisis estadísticos de los datos son del tipo no-paramétrico. 
Cuadro No 10

Test de bondad de ajuste a la curva normal de Kolmogorov-Smirnov

\begin{tabular}{lccc}
\hline Sub-test & Media & D. E. & K-S Z \\
\hline $\begin{array}{l}\text { Comprensión } \\
\text { literal }\end{array}$ & 8.17 & 1.67 & $4.18^{* * *}$ \\
$\begin{array}{l}\text { Comprensión } \\
\text { inferencial }\end{array}$ & 11.20 & 5.22 & $3.01^{* * *}$ \\
$\begin{array}{l}\text { Comprensión } \\
\text { de lectura }\end{array}$ & 19.37 & 6.59 & $3.24^{* * *}$ \\
$\begin{array}{l}\text { Velocidad } \\
\text { lectora }\end{array}$ & 42.81 & 20.86 & $6.10^{* * *}$ \\
\hline
\end{tabular}

*** $\mathrm{p}<.001$

$\mathrm{N}=541$

\section{Análisis comparativos}

El contraste entre los alumnos provenientes de colegios particulares y colegios estatales en los niveles de comprensión de lectura fue realizado a través de la prueba $\mathrm{U}$ de MannWhitney, observándose en el cuadro $\mathrm{N}^{\circ}$ 11 que existen diferencias estadísticas significativas en la comprensión literal $(Z=-16.75, p<.001)$, la comprensión inferencial $(Z=-17.69, \mathrm{p}<.001)$ y la comprensión de lectura $(Z=-17.70, p<$ .001), notándose que en todos los casos los alumnos provenientes de colegios de gestión particular alcanzan mejores niveles de rendimiento que los alumnos provenientes de gestión estatal.

El contraste entre los alumnos provenientes de colegios particulares y los alumnos de colegios estatales respecto de la velocidad de lectura fue realizado también a través de la prueba $U$ de Mann-Whitney, notándose en el cuadro $\mathrm{N}^{\mathrm{o}} 12$ que existen diferencias estadísti-

\section{Cuadro $\mathrm{N}^{\circ} 11$}

Prueba U de Mann-Whitney de la comprensión de lectura según el tipo de gestión del colegio

\begin{tabular}{lrrrr}
\hline Variable & Estatal & Particular & & \\
& $\mathrm{N}=402$ & $\mathrm{~N}=139$ & $\mathrm{U}$ & \multicolumn{1}{c}{$\mathrm{Z}$} \\
\hline Comprensión literal & 206.28 & 458.19 & 1920.00 & $-16.75^{* * *}$ \\
Comprensión inferencial & 200.50 & 473.00 & 80452.30 & $-17.69^{* * *}$ \\
Comprensión de lectura & 201.50 & 472.00 & 81003.00 & $-17.70^{* * *}$ \\
\hline
\end{tabular}

$$
{ }^{* * *} p<.001
$$

\section{Cuadro № 12}

Prueba U de Mann-Whitney de la velocidad lectora según el tipo de gestión del colegio

\begin{tabular}{lcccc}
\hline Variable & $\begin{array}{c}\text { Estatal } \\
\mathrm{N}=402 \\
\text { MR }\end{array}$ & $\begin{array}{c}\text { Particular } \\
\mathrm{N}=139 \\
\text { MR }\end{array}$ & U & $\mathrm{Z}$ \\
\hline Velocidad lectora & 216.95 & 427.32 & 6211.00 & $-13.76^{* * *}$ \\
& & & & \\
\hline
\end{tabular}


cas significativas en la velocidad lectora $(Z=-13.76, p<.001)$, notándose que los alumnos provenientes de colegios de gestión particular alcanzan mejores niveles de rendimiento en la velocidad lectora que los alumnos provenientes de gestión estatal.

El análisis de las relaciones entre la comprensión lectora y la velocidad lectora, presentada en el cuadro $\mathrm{N}^{\mathrm{o}} 13$, permite apreciar que las correlaciones obtenidas entre la comprensión literal y la velocidad lectora $(\mathrm{r}=0.73, \mathrm{p}<.001)$, la relación entre la comprensión inferencial y la velocidad lectora $(r=0.79$, $\mathrm{p}<.001)$ y entre la comprensión de lectura y la velocidad lectora $(\mathrm{r}=0.81$, $\mathrm{p}<.001$ ). Todas son significativas positivas y pueden ser clasificadas como muy altas.

\begin{tabular}{|c|c|}
\hline \multicolumn{2}{|c|}{$\begin{array}{l}\text { Cuadro } \mathrm{N}^{\circ} 13 \\
\text { Correlaciones de Spearman entre } \\
\text { la comprensión de lectura y la } \\
\text { velocidad lectora de toda la muestra }\end{array}$} \\
\hline Correlación & r. \\
\hline $\begin{array}{l}\text { Comprensión literal - } \\
\text { velocidad lectora }\end{array}$ & $0.73 * * *$ \\
\hline $\begin{array}{l}\text { Comprensión inferencial - } \\
\text { velocidad lectora }\end{array}$ & $0.79 * * \star$ \\
\hline $\begin{array}{l}\text { Comprensión de lectura - } \\
\text { velocidad lectora }\end{array}$ & $0.81^{* * *}$ \\
\hline
\end{tabular}

La comparación de las relaciones entre comprensión de lectura y velocidad lectora según el tipo de colegio del cual provienen los alumnos (cuadro $\mathrm{N}^{\circ} 14$ ) permite notar que existen diferencias es- tadísticas significativas en la relación entre comprensión literal y velocidad lectora $(Z=3.93, p<.001)$, entre la comprensión inferencial y la velocidad lectora $(Z=4.33, p<.001)$ y entre la comprensión de lectura y la velocidad lectora $(\mathrm{Z}=5.24, \mathrm{p}<.001)$, notándose que en todos los casos las correlaciones más elevadas corresponden a las obtenidas en los alumnos provenientes de colegios de gestión particular respecto de los alumnos provenientes de colegios de gestión estatal.

\section{Cuadro $\mathrm{N}^{\circ} 14$}

Prueba $Z$ de diferencia de correlaciones entre la comprensión de lectura y la velocidad lectora según el tipo de gestión del colegio

\begin{tabular}{lccc}
\hline Correlación & $\begin{array}{c}\text { Estatal } \\
\mathrm{N}=402 \\
\mathrm{r} .\end{array}$ & $\begin{array}{c}\text { Particular } \\
\mathrm{N}=139 \\
\mathrm{r} .\end{array}$ \\
\hline $\begin{array}{l}\text { Comprensión literal - } \\
\text { velocidad lectora }\end{array}$ & $0.63^{* * *}$ & $0.81^{* * *}$ & $3.93^{* * *}$ \\
$\begin{array}{l}\text { Comprensión inferencial- } \\
\text { velocidad lectora }\end{array}$ & $0.55^{* * *}$ & $0.78^{* * *}$ & $4.33^{* * *}$ \\
$\begin{array}{l}\text { Comprensión de lectura- } \\
\text { velocidad lectora }\end{array}$ & $0.67^{* * *}$ & $0.87^{* * *}$ & $5.24^{* * *}$ \\
\hline
\end{tabular}

${ }^{* * *} p<.001$

\section{Análisis complementarios}

El análisis de las diferencias entre correlaciones por género, presentado en el cuadro $\mathrm{N}^{\circ} 15$, permite apreciar que no existen diferencias estadísticas significativas en la comparación de las correlaciones obtenidas entre la comprensión literal y la velocidad lectora $(Z=0.60, p>.05)$, entre la compren- 


\section{Cuadro $\mathrm{N}^{\circ} 15$}

Prueba $Z$ de diferencia de correlaciones entre la comprensión de lectura y la velocidad lectora por género

\begin{tabular}{lccc}
\hline Correlación & Masculino & Femenino & \\
& $\mathrm{N}=272$ & $\mathrm{~N}=269$ & $\mathrm{Z}$ \\
& $\mathrm{r}$. & $\mathrm{r}$. & \\
\hline Comprensión literal - velocidad lectora & $0.74^{* * *}$ & $0.71^{* * *}$ & 0.60 \\
Comprensión inferencial - velocidad lectora & $0.79^{* * *}$ & $0.80^{* * *}$ & 0.30 \\
Comprensión de lectura - velocidad lectora & $0.81^{* * *}$ & $0.82^{* * *}$ & 0.40 \\
& & & \\
\hline
\end{tabular}

${ }^{\star \star *} p<.001$

sión inferencial y la velocidad lectora $(\mathrm{Z}=0.30, \mathrm{p}>.05)$ y entre la comprensión de lectura y la velocidad lectora $(Z$ $=0.40, \mathrm{p}>.05)$. También se puede apreciar que en todos los casos las correlaciones son positivas y fluctúan entre 0.71 y 0.82 , por lo que se puede clasificar como muy altas.

En lo que concierne a la comparación de las variables estudiadas por género (cuadro $\mathrm{N}^{\mathrm{o}} 16$ ) no existen diferencias estadísticas significativas en la com- prensión literal $(\mathrm{Z}=-1.80, \mathrm{p}>.05)$, la comprensión inferencial $(Z=-0.75, p>$ $.05)$, la comprensión de lectura $(Z=-$ $0.22, p>.05)$ y la velocidad lectora $(Z$ $=-0.19, p>.05)$, por lo que se puede concluir que estas variables no se diferencian según el género de los participantes.

En lo que respecta a la comparación de las variables estudiadas según la edad de los participantes (cuadro $\mathrm{N}^{\circ}$ 17) existen diferencias significativas

\section{Cuadro $\mathrm{N}^{\circ} 16$ \\ Prueba U de Mann-Whitney de la comprensión de lectura y la velocidad lectora según género}

\begin{tabular}{lcccc}
\hline Variable & $\begin{array}{c}\text { Masculino } \\
\mathrm{N}=272 \\
\mathrm{MR}\end{array}$ & $\begin{array}{c}\text { Femenino } \\
\mathrm{N}=269 \\
\mathrm{MR}\end{array}$ & $\mathrm{U}$ & $\mathrm{Z}$ \\
\hline Comprensión literal & 263.96 & 278.12 & 34670 & -1.08 \\
Comprensión inferencial & 275.97 & 265.97 & 35231 & -0.75 \\
Comprensión de lectura & 272.47 & 269.51 & 36184 & -0.22 \\
Velocidad lectora & 269.72 & 272.29 & 36237 & -0.19 \\
\hline
\end{tabular}

$* p<.05$ 
Cuadro $\mathrm{N}^{\circ} 17$

Análisis de varianza por rangos de Kruskal-Wallis de la comprensión de lectura y la velocidad lectora según la edad de los alumnos

\begin{tabular}{lcccc}
\hline Variable & $\begin{array}{c}11 \text { años } \\
\mathrm{N}=228 \\
\mathrm{MR}\end{array}$ & $\begin{array}{c}12 \text { años } \\
\mathrm{N}=248 \\
\mathrm{MR}\end{array}$ & $\begin{array}{c}13 \text { años } \\
\mathrm{N}=65 \\
\mathrm{MR}\end{array}$ & $\mathrm{X}^{2}$ \\
& $353.55_{\mathrm{ab}}$ & $194.56_{\mathrm{ac}}$ & $273.11_{\mathrm{bc}}$ & $128.59^{* * *}$ \\
\hline Comprensión literal & $357.22 \mathrm{ab}$ & $194.96_{\mathrm{ac}}$ & $258.68_{\mathrm{bc}}$ & $129.90^{* * *}$ \\
Comprensión inferencial & $360.15_{\mathrm{ab}}$ & $190.60_{\mathrm{ac}}$ & $265.05_{\mathrm{bc}}$ & $141.61^{* * *}$ \\
Comprensión de lectura & $345.48_{\mathrm{ab}}$ & $203.67_{\mathrm{ac}}$ & $266.67_{\mathrm{bc}}$ & $99.01^{* * *}$ \\
Velocidad lectora & & & &
\end{tabular}

${ }^{*} p<.05^{* *} p<.01{ }^{* * *} p<.001$

Pares de diferencias significativas a través de la $U$ de Mann-Whitney: $a, b, c$

\begin{abstract}
Cuadro $\mathrm{N}^{\circ} 18$
Análisis de varianza por rangos de Kruskal-Wallis de la comprensión de lectura y la velocidad lectora según los tipos de gestión de los colegios de procedencia y el género de los alumnos
\end{abstract}

\begin{tabular}{lccccc}
\hline Variable & $\begin{array}{c}\text { Estatal } \\
\text { Hombres } \\
\mathrm{N}=202\end{array}$ & $\begin{array}{c}\text { Estatal } \\
\text { Mujeres } \\
\mathrm{N}=200 \\
\mathrm{MR}\end{array}$ & $\begin{array}{c}\text { Particular } \\
\text { Hombres } \\
\mathrm{N}=70\end{array}$ & $\begin{array}{c}\text { Particular } \\
\text { Mujeres } \\
\mathrm{N}=69\end{array}$ & $\mathrm{X}^{2}$ \\
& $196.65_{\mathrm{ab}}$ & $216.00_{\mathrm{cd}}$ & $458.21_{\mathrm{ac}}$ & $458.16_{\mathrm{bd}}$ & $282.25^{* * *}$ \\
\hline Comprensión literal & $206.60_{\mathrm{ab}}$ & $196.35_{\mathrm{cd}}$ & $476.18_{\mathrm{ac}}$ & $467.76_{\mathrm{bd}}$ & $313.34^{* * *}$ \\
Comprensión inferencial & $201.87_{\mathrm{ab}}$ & $201.12_{\mathrm{cd}}$ & $476.19_{\mathrm{ac}}$ & $467.75_{\mathrm{bd}}$ & $313.27^{* * *}$ \\
Comprensión de lectura & $215.49_{\mathrm{ab}}$ & $218.43_{\mathrm{cd}}$ & $426.23_{\mathrm{ac}}$ & $428.42_{\mathrm{bd}}$ & $189.37^{* * *}$ \\
Velocidad lectora & & & & & \\
\hline
\end{tabular}

${ }^{*} p<.05^{* *} p<.01{ }^{* * *} p<.001$

Pares de diferencias significativas a través de la $U$ de Mann-Whitney: $a, b, c$ y d

en el caso de la comprensión literal $\left(X^{2}\right.$ $=128.59, \mathrm{p}<.001)$, la comprensión inferencial $\left(X^{2}=129.90, \mathrm{p}<.001\right)$, la comprensión de lectura $\left(X^{2}=141.61, \mathrm{p}\right.$ $<.001)$ y la velocidad lectora $\left(X^{2}=\right.$ 99.01, $\mathrm{p}<.001)$. Además se aprecia que en todos los casos se encuentra que los alumnos de 11 años superan a los de 12 y 13 años y también los alumnos de 13 años superan a los de 12 años.

En la comparación de las variables estudiadas según los tipos de gestión de los colegios de procedencia y el género de los alumnos (cuadro $\mathrm{N}^{\circ} 18$ ) se encuentra que existen diferencias significativas en el caso de la comprensión literal $\left(X^{2}=282.55, \mathrm{p}<.001\right)$, comprensión inferencial $\left(X^{2}=313.34, \mathrm{p}<\right.$ $.001)$, comprensión de lectura $\left(X^{2}=\right.$ $313.27, \mathrm{p}<.001)$ y velocidad lectora $\left(X^{2}=189.37, \mathrm{p}<.001\right)$. También se encuentra que en todos los casos los alumnos procedentes de colegios con gestión particular, ya sean hombres o 
mujeres, superan a los procedentes de colegios de gestión estatal, ya sean hombres o mujeres.

Para realizar la comparación entre la comprensión literal y la comparación inferencial, en primer lugar se procedió a transformar ambas puntuaciones en un sistema métrico común, por ello previamente se dividió el total del puntaje literal entre el número total de ítemes (10) y el puntaje inferencial entre el número total de ítemes (18). Una vez que ambas puntuaciones fueron transformadas, se procedió a calcular la prueba no paramétrica de Wilcoxon para grupos relacionados.

Los resultados presentados en el cuadro $\mathrm{N}^{\circ} 19$ permiten observar que en toda la muestra existen diferencias estadísticas significativas $(\mathrm{Z}=17.25, \mathrm{p}<$ .0001), notándose que la comprensión literal alcanza una mayor media de ran- gos $($ M.R. $=237.28)$ que la comprensión inferencial (M.R. $=141.96$ ).

En el caso de la comparación sólo de los colegios estatales, existen diferencias estadísticas significativas $(Z=16.46)$, apreciándose que la comprensión literal (M.R. = 207.86) supera a la comprensión inferencial (M.R. = 85.13).

En el contraste de los colegios particulares existen diferencias estadísticas significativas $(Z=-5.76)$, pero en este caso la comprensión inferencial (M.R. $=60.50)$ supera a la comprensión literal (M.R. $=29.50)$.

Los resultados del contraste en los hombres indican que existen diferencias estadísticas significativas $(\mathrm{Z}=$ 11.74), notándose que la comprensión literal (M.R. $=118.81)$ supera a la comprensión inferencial (M.R. $=72.92$ ).

Finalmente, en la comparación en las mujeres también existen diferencias es-

\section{Cuadro No 19}

Análisis comparativo entre la comprensión literal y la comprensión inferencial a través de la prueba de Wilcoxon para grupos relacionados

\begin{tabular}{lccc}
\hline Grupo comparado & $\begin{array}{c}\text { Comprensión literal } \\
\text { M.R. }\end{array}$ & $\begin{array}{c}\text { Comprensión inferencial } \\
\text { M.R. }\end{array}$ & Z \\
\hline $\begin{array}{l}\text { Toda la muestra } \\
\mathrm{N}=451\end{array}$ & 237.28 & 141.96 & $17.25^{* * *}$ \\
$\begin{array}{l}\text { Colegio estatal } \\
\mathrm{N}=402\end{array}$ & 207.86 & 85.13 & $16.46^{* * *}$ \\
$\begin{array}{l}\text { Colegio particular } \\
\mathrm{N}=139\end{array}$ & 29.50 & 60.50 & $-5.76^{* * *}$ \\
$\begin{array}{l}\text { Varones } \\
\mathrm{N}=272\end{array}$ & 118.81 & & \\
Mujeres & & 72.92 & $11.74^{* * *}$ \\
$\mathrm{~N}=269$ & 119.10 & 64.78 & $12.68^{* * *}$ \\
\hline
\end{tabular}

${ }^{* * *} p<.0001$ 
tadísticas significativas $(Z=12.68)$, notándose que también la comprensión literal (M.R. = 119.10) supera a la comprensión inferencial (M.R. $=64.78)$.

\section{DisCUSIÓN}

Los resultados psicométricos obtenidos en la prueba de comprensión lectora de sexto grado (PCL-6) indican que el instrumento desarrollado por Carreño (2000) para evaluar la comprensión lectora cumple con los requisitos básicos, pues la confiabilidad por consistencia interna alcanza un coeficiente Kuder Richardson 20 de 0.94 en la prueba completa, 0.80 en la primera parte, y 0.90 en la segunda parte. El estudio de la validez de constructo, realizado sobre la base del análisis factorial confirmatorio, permite demostrar que las comprensiones literales e inferenciales incluidas en el instrumento constituyen una sola dimensión, la cual correspondería a la comprensión de lectura. Estos hallazgos corroboran que la prueba es válida y confiable.

En relación con la prueba de velocidad lectora (PVL), desarrollada por Espada (2000), los hallazgos obtenidos en los análisis psicométricos realizados señalan que el instrumento presenta confiabilidad en términos de estabilidad, pues los resultados del método test-retest indican que se alcanza un coeficiente de correlación de Pearson de 0.89 , lo que permite concluir que la prueba permite obtener puntajes confiables en el tiempo. El estudio de la validez de contenido alcanza un coeficiente V de Aiken de 0.95. Estos resultados confirman que el instrumento presenta validez y confiabilidad.

El resultado que permite apreciar un mejor desempeño en los alumnos provenientes de colegios particulares en los niveles de comprensión de lectura, que en los alumnos de colegios estatales, corresponde a un hallazgo similar al reportado por Carreño en todo el Perú. Este resultado estaría en relación con el tipo de enseñanza que se brinda en los diferentes tipos de colegios según el tipo de gestión que presentan, y pone en relieve el déficit del sistema educativo del Estado respecto de los establecimientos particulares, reflejados en una deficiente enseñanza del alumnado especialmente en lo que se refiere a la lectura y la comprensión.

El análisis más detallado de la comparación entre el tipo de gestión de los colegios corrobora esta tendencia, pues los alumnos que estudian en colegios con gestión particular presentan un mejor rendimiento que los alumnos de colegios con gestión estatal, tanto en comprensión literal como en comprensión inferencial. Este resultado concuerda con los estudios de Sánchez (1987) y Carreño (2000).

La predominancia de los rendimientos en la comprensión literal respecto de la comprensión inferencial en los alumnos en los colegios con gestión estatal indica que los alumnos se ubican en un primer nivel de comprensión que, de 
acuerdo con Van Dijk y Kinsth (Pinzás, 1993), corresponde a la microestructura, no logrando acceder a un nivel más elevado como el de la macroestructura, el cual supone una integración y reintegración de las proposiciones leídas en niveles cada vez más complejos, hasta llegar a un modelo o comprensión global del texto. Tal como lo plantea Johnston (1983), para llegar a este nivel son necesarias las inferencias que le permiten al lector establecer conexiones lógicas entre las proposiciones, a partir de la generación de información que no se encuentra en el texto.

Estos resultados aluden a la importancia de enseñar y estimular las habilidades de comprensión inferencial desde el primer grado, de tal manera que se pueda ir añadiendo a los contenidos literales parte de sus conocimientos previos hasta poder llegar a una comprensión coherente e integrada del texto, tal como lo proponen Linuesa \& Domíguez (1999).

En relación con la velocidad lectora, se aprecia que los alumnos provenientes de colegios particulares presentan mejores niveles de velocidad lectora que los alumnos de colegios estatales. Este hallazgo está en correspondencia con los resultados alcanzados en la comparación de la comprensión de lectura, debido probablemente a que existe un efecto diferencial de la enseñanza que reciben los alumnos según el tipo de gestión que presenta el colegio donde estudian.
En lo que concierne a la relación entre la comprensión de lectura y la velocidad, los hallazgos indican que la comprensión de lectura y la velocidad lectora presentaron una correlación muy alta, positiva y significativa. Este resultado abona en favor de los autores que indican que sí existe relación entre ambos aspectos de la lectura, como es el caso de Gates, que en 1921 determinó que ambas constituyen dos factores distintos pero relacionados, LaBerge \& Samuels (1985), quienes desarrollaron un modelo en el cual ponen énfasis en lo automático de la ejecución de ciertos componentes del proceso de lectura, y Nuttall (1996), quien indica que ambas se encuentran estrechamente relacionadas y que el leer lentamente produce un círculo vicioso en los lectores deficientes, pues cuando los lectores no entienden un material a menudo tienden a disminuir la tasa de lectura y, por lo tanto, el gusto por leer porque les toma mucho tiempo, lo cual genera como resultado final que este tipo de lectores lean poco, manteniéndose así el círculo vicioso. Además, Nuttall (1996) sugiere que si se aumentan las proporciones de lectura, el lector puede entrar en el círculo del buen lector, es decir que, al leer más rápidamente, se puede animar, y, con más lecturas, la comprensión tiende a mejorar. Esta concepción de Nuttall (1996) se sustenta en la propuesta de Stanovich (1980), quien alude a un fenómeno identificado por Merton en 1968 (Ber- 
mejo, 1994) como el "efecto de Matthew", el cual sugiere que los estudiantes que más leen aumentan sus habilidades de lectura.

El hecho de que los alumnos provenientes de colegios particulares presentaron una mayor relación entre la comprensión de lectura y la velocidad lectora que los provenientes de colegios estatales indica que el efecto diferencial de la enseñanza, observado en los hallazgos previos, se corrobora en este aspecto, donde se estudia la relación comparativa de los aspectos estudiados y que, de acuerdo con lo propuesto por Nuttall (1996), en los colegios estatales se presenta de forma aún muy baja el "efecto de Matthew".

En relación con la comparación de la lectura por género, los resultados indican que no existen diferencias significativas, estos hallazgos son contrarios a lo propuesto por Anderson (1993), quien dice que se espera que los hombres en situaciones favorables tienden a obtener mejores desempeños en la comprensión de lectura que las mujeres. Más bien reflejarían que la fuente más consistente de diferenciación es la enseñanza recibida, expresada por el tipo de gestión del colegio de procedencia.

Respecto de la variable edad, llama la atención la gran diferencia encontrada en la comprensión de lectura en un mismo grado. Estos hallazgos coinciden con los alcanzados por Carreño (2000), quien encuentra que los niños de 11 años tienen un mejor desempeño que los de 12 y 13 años. Tal como indica Carreño (2000), es posible que los niños de 12 y 13 años tengan dos o más años de atraso en su escolaridad, lo cual tendría un efecto negativo sobre su desempeño en la comprensión lectora.

En líneas generales los resultados muestran la gran heterogeneidad de la población escolar, especialmente en lo que se refiere a la comprensión de lectura y la velocidad lectora. La revisión de los resultados de la velocidad lectora indican que en términos generales los alumnos presentan bajos niveles de rendimiento en relación con la clasificación propuesta por Fry (1975), las propuestas de Jensen (1986), Higgins \& Wallace (1989), Dubin \& Bycina (1991) y Nuttall (1996), así como los resultados alcanzados en alumnos españoles en el estudio de Espada (2000).

Estos resultados negativos para la velocidad lectora son más dramáticos al compararse por el tipo de gestión del colegio de procedencia, pues los colegios estatales son marcadamente deficitarios en la velocidad lectora respecto de los colegios particulares, lo cual lleva a la imperiosa necesidad de romper el círculo vicioso del mal lector del cual habla Nuttall (1996).

Finalmente, se puede concluir que los resultados observados en el presente estudio concuerdan con los hallazgos de las investigaciones realizadas en la última década en el Perú, como es el caso de Thorne (1991), Pinzás (1993), 
Nakano (1996), Morales (1997) y Carreño (2000), en las que se aprecia un bajo desarrollo de las habilidades lectoras de los niños peruanos en general, lo cual indica que el sistema educativo a lo largo de la educación primaria no permite a los alumnos llegar a desarrollar estas habilidades, por lo cual es indispensable tratar de revertir esta situación con el fin de lograr un desarrollo educativo que permita responder adecuadamente a las demandas de una cultura globalizada y caracterizada como una sociedad del conocimiento.

\section{REFERENCIAS}

Alarcón, R. (1991). Métodos y diseños de investigación del comportamiento. Lima: Universidad Peruana Cayetano Heredia.

Alegría, J. (1991). Mecanismos especificos de la lectura. Buenos Aires: Editorial Paidós.

Alliende F., Condemarín, M. \& Milicic, N. (1993). Prueba CLP formas paralelas. Manual para la aplicación de la prueba de comprensión lectora de complejidad lingüística progresiva: 8 niveles de lectura. Santiago de Chile: Ediciones Universidad Católica de Chile.

Alliende, F. \& Condemarín, M. (1994). La lectura: teoría, evaluación y desarrollo. Santiago de Chile: Editorial Andrés Bello.

Anastasi, A. (1986). Evolving concepts of test validation. Anual Review of Psychology 37, 1-15.

Anastasi, A. \& Urbina, S. (1998). Tests psicológicos. México: Editorial Prentice Hall.

Anderson, J. (1980). Desde niñas: Género $y$ postergación en el Perú. Lima: Unicef/Consorcio Mujer.

Babbie, E. (2000). Fundamentos de la investigación social. México: International Thomson Editores.

Bermejo, V. (1994). Desarrollo cognitivo. Madrid: Síntesis.

Brown, F. (1996). Principios de la medición en psicología y educación. México: Editorial El Manual Moderno.

Carreño, C.B. (2000). Comprensión de lectura al finalizar primaria en niños peruanos. Inédito. Tesis para obtener el 
grado de maestro. Pontificia Universidad Católica del Perú.

Champeau de López, S. (1993). Developing reading speed. Journal of Reading 3(2), 210-260.

Cuetos, F. (1996). Psicología de la lectura. Madrid: Editorial Escuela Española.

De Vega, M.; Carreiras, M.; GutiérrezCalvo, M. \& Alonso Quecuty, M. (1990). Lectura y comprensión: Una perspectiva cognitiva. Madrid: Alianza Editorial S.A.

Dubin, F. \& Bycina, D. (1991). Academic reading and the ESL/EFL teacher. En: M. Celce-Murcia (Ed.). Teaching english as a second or foreign language. Boston: Heinle and Heinle.

Escurra, L.M. (1988). Cuantificación de la validez de contenido por el criterio de jueces. Revista de Psicología de la Pontificia Universidad Católica del Perú 6(1 y 2), 103-111.

Espada J. (2000). Programa para mejorar la competencia lectora, velocidad, precisión y entonación. Andalucía: Consejería de Educación y Ciencia de la Junta de Andalucía.

Fry, E. (1975). Reading drills for speed and comprehension. Providence R.I.: Jamestown Publishers.

Gates, A. (1921). An experimental and statistical study of reading and reading tests. Journal of Educational Psychology 12, 303-314.

Gibson, E.J. (1973). Trends in perceptual development: implications for the reading process. En: A.D. Pick (Ed.). Minnesota symposia on child psychology. Vol. 8. Minneapolis: University of Minnesota Press.
Gonzales, V.R. (1998). Comprensión lectora en estudiantes universitarios iniciales. Persona 1, 43-65.

Goodwin, C.J. (1998). Research in psychology: methods and design. New York: John Wiley y Sons, In.

Graziano, A.M. \& Raulin, M.L. (1989). Research methods, a process of inquiry. Albany: Harper Collins Publishers.

Greaney, V. (1996). Promoting reading in developing countries. Washington: International Reading Association.

Hernández, S.R., Fernández, C.C. \& Baptista, L.P. (1998). Metodología de la investigación. México: McGraw-Hill.

Higgins, J. \& Wallace, R. (1989). Hopalong: A computer reader pacer. System 17(3), 389-399.

Jensen, L. (1986). Advanced reading skills in a comprehensive course. En: F. Dubin, D. Eskey \& W. Grabe (Eds.). Teaching second language reading for academic purpose. Reading M.A.: Addison-Wesley Publishing.

Johnston, P. (1983). Reading comprehension assessment: a cognitive basis. Newark: International Reading Association.

LaBerge, D. \& Samuels, S.J. (1985). Toward a theory of automatic information processing in reading. En: H. Singer \& R.B. Ruddell (Eds.). Theoretical models and processes of reading. Newark Del.: International Reading Association.

Linuesa, M. \& Domíguez (1999). La enseñanza de la lectura, enfoque psicolingüístico y sociocultural. Madrid: Editorial Pirámide. 
Lohr, S. (1999). Muestreo: diseño y análisis. México: International Thomson Editores.

Molina, S. (1991). Psicopedagogía de la lectura. Madrid: CEPE.

Morales, S. (1997). Comprensión de lectura en niños de los grados $4^{\circ}$ y $5^{\circ}$ de primaria de Lima. Análisis de inferencias y metacognición. Inédito. Tesis de licenciatura. Pontificia Universidad Católica del Perú.

Morales, A. (1997). El mejoramiento de la capacidad para aprender a través de la lectura. Revista de Psicología de la Pontificia Universidad Católica del Perú 15(2), 253-277.

Nakano, T. (1996). El programa curricular de articulación inicial-primer grado y el rendimiento de la prueba de comprensión de lectura en niños de primer grado. Inédito. Tesis de licenciatura en psicología. Pontificia Universidad Católica del Perú.

Neisser, U. (1977). Cognition and reality. San Francisco: W.H. Freeman Company.

Nicasio García, J. (1995). Manual de dificultades de aprendizaje: Lenguaje, lecto-escritura y matemáticas. Madrid: Editorial Narcea.

Nieto, M. (1980). Estudio de la dislexia. Buenos Aires: Editorial Paidós.

Noriega, E. (1998). Estudio cualitativo de los niveles de comprensión lectora de un grupo de niños deficientes y buenos lectores antes y después de un programa de intervención. Revista de Psicología de la Facultad de Psicología de la Universidad Nacional Mayor de San Marcos 2(2), 159-171.
Nuttall, C. (1996). Teaching reading skills in a foreign language. Oxford: Heinemann English Language Teaching.

Pérez, C. (2000). Técnicas de muestreo estadístico: Teoría, práctica y aplicaciones informáticas. México: Alfaomega grupo editor S.A. de C.V.

Pinzás, J. R. (1986). Del símbolo al significado. El caso de la comprensión de lectura. Revista de Psicología de la Pontificia Universidad Católica del Perú 4(1), 4-13.

Pinzás, J.R. (1987). Rol de los padres en el aprendizaje de la lectura y la comprensión de la lectura. Revista de Psicología de la Pontificia Universidad Católica del Perú 5(2), 177-188.

Pinzás, J.R. (1993). A cognitive monitoring in reading comprehension, a study of difference among schools in Lima. Tesis doctoral, K.U. Nijmegen.

Pinzás, J.R. (1995). Leer pensando. Lima: Asociación de investigación aplicada y extensión pedagógica Sofía Pinzás.

Pinzás, J.R. (1997). Metacognición y lectura. Lima: Pontificia Universidad Católica del Perú.

Puente, A. (1991). Comprensión de la lectura y acción docente. Madrid: Editorial Pirámide.

Rinaldi, R. \& Sola, M. (1994). Modelo hegemónico de la práctica de la lectura en $3^{\circ}$ y $4^{\circ}$ : Implicancias en la conducta lectora infantil. Lectura y vida 14(4), 2328.

Rosenberg, K.M. \& Daly, H.B. (1993). Foundations of behavioral research: A basic question approach. Fort Worth: Harcourt Bruce Jovanovich College Publishers. 
Rosnow, R.L \& Rosenthal, R.L. (1996). Beginning behavioral research a conceptual primer. New Jersey: PrenticeHall Inc.

Rumelhart, D.E. (1977). Toward interactive model of reading. En: S. Dornic (Ed.). Attention and performance VI. Hillsdale, New Jersey: Lawrence Erlbaum Associates.

Salkind, N. J. (2000). Métodos de investigación. México: Prentice-Hall Hispanoamericana S.A.

Salvia, J. \& Ysseldike, J.E. (1998). Assesment. Boston: Houghton \& Mifflin Company.

Samuels, J. \& Kamil, A. (1988). Models of the reading process. En: P.D. Pearson, P. Mosenthal, M. Kamil \& R. Barr (Eds.). Handbook of Reading Research. New York: Longman, Inc.

Sánchez, H. (1987). Lectura: investigaciones en el Perú. Lima: Inide.

Sánchez, H. \& Reyes, C. (1998). Metodología y diseños en la investigación cientifica. Lima: Editorial Mantaro.

Smith, F. (1978). Reading without nonsense. Columbia: Teachers College Press.

Sole, I. (1998). Estrategia de lectura. Barcelona: Instituto de Ciencias de la Comunicación.

Stanovich, K. (1980). Toward an interactive-comprehension model of individual difference the developmental of reading fluency. Reading research quarterly 16, 32-71.

Tapia, V. (1999). Efectos de un programa de estrategias cognitivas y metacognitivas para mejorar la comprensión lectora. Revista de Psicología de la Facultad de Psicología de la Universidad Nacio- nal Mayor de San Marcos 3(4), 9-21.

Thorne, C. (1991). A study of beginning reading in Lima. Tesis doctoral. K.U. Nijmegen.

Thorne, C. \& Pinzás, J. (1988). "Factor affecting reading achievement in Perú". Ponencia presentada en la conferencia anual de la American Educational Research Association.

Vidal-Abarca, E. \& Gilabert, R. (1991). Comprender para aprender: un programa para mejorar la comprensión y el aprendizaje de textos. Madrid: CEPE. 


\section{Anexo \\ Prueba de comprensión lectora para el sexto grado PCL-6}

\section{Texto 1}

Daniel era un niño de nueve años que vivía con sus padres. Al frente de su casa había un parque verde, en el que jugaba con sus amigos Pedro, Luis y Juan. A ellos les gustaba jugar fútbol en la canchita del parque. Pero algunas veces la plataforma de cemento estaba ocupada y jugaban sobre el jardín. Cuando hacían eso, frecuentemente aparecía un hombre de pelo blanco que se dirigía hacia ellos.

—¿Qué hacen en el césped? ¡Salgan de allí! —decía molesto-.

-Sólo estamos jugando. No nos moleste — decían los niños.

—Pero miren las plantas... ¡Están pateando las plantas y las flores! ¡Váyanse!

Los niños salían corriendo del lugar, no sin antes burlarse y reírse del viejo.

Un día Daniel se despertó muy temprano. Todos en su casa aún dormían. Como ya no tenía sueño, abrió su ventana para ver como amanecía. Estaba un poco oscuro aún. Entonces divisó a un hombre en el parque.

— ¿Tan temprano sale ese señor a jugar al parque? —se preguntó. Luego se percató de que el hombre no estaba jugando. Era el viejo que siempre lo botaba del parque. Había puesto una larga manguera y regaba el jardín. Luego sacó la hierba mala. Más tarde podó las plantas y arbolitos con unas tijeras. El hombre sudaba, pero se veía feliz. Luego apareció una mujer con unas largas trenzas que silbaba. Ella le secó el sudor con una toalla pequeña. Después le dio algo de comer y beber.

-Pobre hombre, parece que recién está tomando desayuno — pensó el niño—. Ha estado trabajando sin tener nada en el estómago-. En ese instante Daniel escuchó que su madre lo llamaba para tomar desayuno y cerró la ventana.

Por la tarde, Daniel observó por la ventana nuevamente, y el señor seguía en el parque. Estaba removiendo la tierra. Daniel lo vio irse recién cuando se ocultó el sol.

—Ayer también lo vi irse a esa hora —pensó.

Al día siguiente, Pedro, Luis y Juan le dijeron a Daniel para ir a jugar al parque. —Sólo si encontramos la cancha vacía. Así respetamos el esfuerzo de una persona-. Los amigos no lo entendieron pero aceptaron la idea.

1. ¿Cómo se llamaba el niño del cuento?
a. Juan.
b. Pedro.
c. Daniel.

2. ¿Cuántos años tenía el niño?

a. 9 años.

b. 8 años.

c. 10 años.

3. ¿Qué les gustaba jugar a los niños?

a. A la escondidas.

b. A patear las plantas y las flores.

c. Fútbol. 
4. ¿Qué hacía el viejo en el parque?
a. Jugaba fútbol.
b. Dormía.
c. Regaba y podaba las plantas.

5. ¿Quién le llevó comida y bebida al viejo?
a. Daniel.
b. Una mujer.
c. Luis y Pedro.

6. ¿Cómo se le veía al viejo cuando trabajaba en el jardín?
a. Feliz.
b. Molesto.
c. Aburrido.

7. ¿Cómo era el parque frente a la casa de Daniel?
a. Estaba bien cuidado.
b. Estaba sucio.
c. Siempre estaba mojado.

8. ¿Qué era la plataforma de cemento?
a. La casa del viejo.
b. La vereda.
c. La cancha de fútbol.

9. ¿A qué hora empezaba el viejo a trabajar?
a. Al ocultarse el sol.
b. Al amanecer.
c. Al mediodía.

10. ¿Qué significa "Daniel divisó a alguien"?
a. Daniel vio a alguien.
b. Daniel gritó a alguien.
c. Daniel avisó a alguien.

11. ¿Qué quiere decir "Daniel se percató..."?
a. Daniel se alegró.
b. Daniel se despertó.
c. Daniel se dio cuenta.

12. ¿Por qué Daniel al final sólo quiere jugar en la cancha vacía?
a. Porque le tenía miedo al viejo.
b. Porque no quería destruir el parque.
c. Porque se juega mejor allí.

13. ¿Cómo era el viejo del parque?
a. Un señor que quería las plantas y las flores.
b. Un señor que odiaba el fútbol y los niños.
c. Un hombre de mal humor. 


\section{Escurra}

14. ¿Cómo era la mujer que le llevó algo de comer al viejo del parque?
a. Alegre y cariñosa.
b. Le gusta comer.
c. Conversadora y despeinada.

15. ¿Cuál es el mejor título para la historia?
a. El fútbol.
b. Los amigos de Daniel.
c. El jardinero.

\section{Texto 2}

Tomás subió al ómnibus con su padre. Eran las tres de la tarde y el sol brillaba. Poco a poco se fueron alejando de los grandes edificios. Así empezó el viaje hacia "Pasos". En ese pueblo había nacido la madre de Tomás. Él quería viajar desde antes de su cumpleaños, pero recién pudo visitar el lugar después de su cumpleaños. El niño estaba contento y muy inquieto.

— ¡Voy a conocer la casa donde mi mamá vivió de chiquita! — decía.

— iTe va a encantar! Pero no te muevas mucho — le advirtió su padre. —Cuando uno viaja a lugares ubicados en altura, debe andar con calma. Así le damos tiempo al cuerpo para que se acostumbre.

Después de bordear el mar, empezaron a subir por la montaña. Poco a poco el paisaje fue cambiando. En la montaña, todo estaba cubierto de plantas variadas y flores de colores. Por unos minutos pararon en la oficina de control vial. Tomás y su padre bajaron del ómnibus un momento. El niño corrió un poco y respiró el aire de la montaña. Luego recolectó algunas flores.

—Son para mamá — dijo contento.

Al volver al ómnibus, Tomás se dio cuenta de que una señora mayor lo observaba. Tenía la mirada triste. El niño se le acercó con una sonrisa y le regaló una flor.

- Seguro que a usted también le gustan las flores, como a mi mamá. A ella le gustan las anaranjadas - dijo Tomás. Luego le contó que era la primera vez que viajaba al pueblo, que su madre los estaba esperando allá y otras cosas más.

Más tarde Tomás comenzó a sentir un dolor de cabeza. Entonces, la viejita le dio un mate de coca para tomar. Así el niño se sintió mejor y se quedó dormido.

Cuando despertó ya había llegado al pueblo. La luz de la luna iluminaba el ambiente. Apenas Tomás vio a su mamá corrió y la abrazó.

Al día siguiente, mientras Tomás y sus padres desayunaban, sonó el timbre de la casa. Al abrir la puerta no había nadie. Pero todos se sorprendieron al ver un gran ramo de flores anaranjadas en una canasta, cerca de la puerta.

— ¿Quién habrá mandado estas lindas rosas? — se preguntaron.

—Leeré la nota — dijo el padre-, dice:

"Para la mamá del niño que me devolvió la sonrisa,

\section{Elisa Montes"}

—Ah, yo la conozco — dijo la mamá-. Es una viejita que se quedó muy sola cuando su hija y su único nieto se tuvieron que ir a vivir a otro país.

1. ¿A dónde viajaban Tomás y su padre?
a. A la montaña.
b. A la playa.
c. A la gran ciudad. 
2. ¿A qué hora partieron Tomás y su padre de viaje?
a. En la mañana.
b. A las tres de la tarde.
c. En la noche.

3. ¿Cómo era la montaña?
a. Con muchas plantas y flores de colores.
b. Con edificios.
c. Con arena y mar.

4. ¿Quién había nacido en el pueblo "Pasos"?
a. La mamá de Tomás.
b. La viejita.
c. Tomás.

5. ¿Qué le regaló Tomás a la viejita?
a. Un dulce.
b. Un mate de coca.
c. Una flor.

6. ¿Cómo era Tomás?
a. Era conversador.
b. Estaba enfermo.
c. Era dormilón.

7. ¿Dónde vivía Tomás?
a. En un pueblito.
b. En la montaña.
c. En la ciudad.

8. ¿Por qué Tomás quería conocer "Pasos"?
a. Porque era su cumpleaños.
b. Porque allí había nacido su mamá.
c. Porque estaba de vacaciones.

9. ¿Por qué a Tomás le dio dolor de cabeza?
a. Porque había estado enfermo.
b. Por haberse movido a mucha altura.
c. Porque extrañaba a su mamá.

10. ¿A qué hora llegaron al pueblo?
a. En la noche.
b. A la hora del desayuno.
c. En la tarde.

11. ¿Quién mandó las rosas anaranjadas?
a. El padre.
b. La viejita del ómnibus.
c. Tomás. 


\section{Escurra}

12. ¿Para quién eran las rosas anaranjadas?
a. Para la familia de Tomás.
b. Para la mamá de Tomás.
c Para Tomás.

13. ¿Por qué la viejita tenía la mirada triste?
a. Porque estaba lejos de sus familiares.
b. Porque no tenía flores.
c. Porque estaba enferma.

\section{Prueba de velocidad lectora}

Leer en voz alta la siguiente lectura lo más rápido que sea posible:

\section{Los castores del río Meldrum}

Mi abuela india recuerda cómo era el río Meldrum cuando era pequeña. En él apagaban su sed rebaños de alces y ciervos, los castores chapoteaban con su cola, las truchas saltaban para coger la mosca de mayo y, a millares, los patos silvestres se atracaban de algas, sus bosques de abetos y sus numerosas presas mantenidas por la colonia de castores. Así lo habían conocido sus padres y los padres de sus padres.

Pero los castores habían desaparecido por el expolio de los cazadores y peleteros y ahora sus aguas estaban estancadas, en algunos trechos el cauce estaba seco y en otros era un riachuelo. Las presas abandonadas de los castores eran un vestigio de cuando éstos pululaban en sus aguas y cuidaban las presas para mantener el nivel de los lagos y ríos y proteger sus madrigueras. Aquí es donde se instalaron mis padres en 1931.

En los bosques y pantanos toda especie se conserva y multiplica alimentándose de otra. Si volvían a llenarse los pantanos del arroyo Meldrum, las semillas y tubérculos que estaban aún enterradas en su cauce servirían de alimento a las ratas almizcleras, aves acuáticas y peces. A cazarlos vendrían el visón, la nutria y otros carniceros. Sólo había que crear un ambiente propicio para unos para que acudiesen los otros.

Ellos solos repararon algunas presas de castores. Hasta que en 1941 el departamento de caza les entregó dos parejas de castores. ¡Esa era la solución! En muchos de sus lagos encontramos aún sus viviendas. A la salida de todos los pantanos, grandes y pequeños, había una presa de castores. Después de medio siglo los castores volvieron al valle del río Meldrum que, en la actualidad, ha recuperado gran parte de su antiguo esplendor. 